\title{
Nationalstaat und Europäische Union: eine Bestandsaufnahme
}

\author{
von Thomas Fehrmann
}

\begin{abstract}
Der nachfolgende Bericht informiert über ein am 30. November 2012 in Salzburg durchgeführtes Kolloquium zum Thema „Nationalstaat und Europäische Union: eine Bestandsaufnahme". Die vom Nomos-Verlag und dem Internationalen Institut für Staatsund Europawissenschaften (ISE), Berlin, getragene Veranstaltung basierte auf vier Panels, in deren Rahmen die Mitherausgeber der ZSE die übergreifende Fragestellung aus der Sicht der Rechts-, Wirtschafts-, Sozial- und Geschichtswissenschaften erörterten. Unter Einbezug des Publikums ergab sich eine interdisziplinäre, international vergleichende und die Grenze zwischen Wissenschaft und Praxis häufig überbrückende Debatte über den Zustand und die Entwicklungsperspektiven der europäischen Integration.
\end{abstract}

This report outlines and summarises the proceedings of a colloquium entitled "The Nation-State and the European Union: Taking Stock", held on 30 November 2012 in Salzburg. The debate, organised by Nomos Publishers and the International Institute for Comparative Government and European Policy (ISE), Berlin, revolved around four panels (public law, economics, social sciences, and history) consisting of members of this journal's Editorial Board. With the active participation of the wider audience, a number of central issues concerning the current state and future development of European integration were subject to an intense interdisciplinary and international comparative discussion that succeeded in bridging the gap between academic and political-administrative discourses.

\section{Einleitung}

Das vom Salzburger Fürstbischof Leopold Anton von Firmian in den Jahren 1736 bis 1740 erbaute Schloss Leopoldskron bot in gleich zweifacher Hinsicht eine historisch eindrucksvolle Kulisse für das hier zu dokumentierende Kolloquium zum Thema „Nationalstaat und Europäische Union: eine Bestandsaufnahme“. Zum einen wurde das Schloss nach dem Erwerb durch Max Reinhardt im Jahr 1918 parallel zu den von ihm mitbegründeten Salzburger Festspielen zu einem zentralen Ort der pan-europäischen Kunst- und Kulturlandschaft; zum anderen entwickelte sich Leopoldskron, zwischenzeitlich von den Nationalsozialisten konfisziert, nach 1945 unter amerikanischer Federführung und mit Zustimmung von Reinhardts Witwe zu einer der ersten Begegnungsstätten für ame- 
rikanische und europäische Studierende, Hochschullehrer und Politiker (von beiden Seiten des Eisernen Vorhangs ${ }^{1}$ ) im Rahmen des noch heute das Schloss betreibenden Salzburg Global Seminar.

Neben dieser genuin europäischen Grundorientierung bot Leopoldskron mit der Parker Hall im Meierhof des Schlosses einen der produktiven Diskussion in jeder Hinsicht zuträglichen Rahmen. Die ganztägige Veranstaltung war Teil eines vom Nomos-Verlag und dem Internationalen Institut für Staats- und Europawissenschaften (ISE), Berlin, aus Anlass der Emeritierung von Joachim Jens Hesse angebotenen Programms, das darüber hinaus ein Kammerkonzert des dem Salzburger Mozarteum entwachsenen „Belmonte-Quartetts“ und ein festliches Abendessen im Venezianischen Zimmer des Schlosses beinhaltete.

Hesse eröffnete die Veranstaltung mit einem kurzen Geleitwort, das von den benannten historischen Besonderheiten des Tagungsorts zu den materiellen Fragestellungen des Kolloquiums überleitete: Welchen Beitrag leisten die die Staats- und Europawissenschaften konstituierenden Disziplinen (also die Rechts-, Wirtschafts-, Sozial- und Geschichts-/Kulturwissenschaften) im Rahmen des fortlaufenden Europäisierungsprozesses und vor allem mit Blick auf die gegenwärtig erkennbaren Krisen in der Eurozone? Wer vertritt die „,Völker Europas“ in angemessener Form und im Rahmen demokratisch legitimierter Verfahren? Und: Kann dem gemeinsamen Wunsch nach europäischer Orientierung nur durch ein „Mehr an Europa“ entsprochen werden? Zur Beantwortung dieser Fragen wurde das Kolloquium in vier aufeinander folgende Panels strukturiert, die mit Mitgliedern des Herausgeberkreises dieser Zeitschrift besetzt waren und diese übergreifenden Fragestellungen aus der Sicht der benannten Disziplinen zu diskutieren suchten. Nach knappen einleitenden statements der Diskussionsleiter waren je zwei materielle Beiträge vorgesehen, die abschließend durch einen discussant zusammengefasst und in die breitere Diskussion überführt wurden. Der nachfolgende Bericht orientiert sich an dieser Gliederung und schließt mit einer kurzen zusammenfassenden Darstellung der Diskussionsergebnisse.

\section{Rechtswissenschaftliches Panel}

In seiner umfassend angelegten und vor allem die rechtspraktische Dimension der gegenwärtigen europapolitischen Herausforderungen hervorhebenden Einleitung skizzierte Peter M. Huber (Karlsruhe/München) die Agenda für das rechts-

1 Vgl. Ryback, T.W.: The Salzburg Global Seminar - A Community of Fellows Salzburg/Washington, D.C., undatiert, www.salzburgglobal.org/current/history-b.cfm. 
wissenschaftliche Panel der Veranstaltung. Er verwies bereits zu Beginn seiner Ausführungen darauf, dass die Beschreibung der Europäischen Union (EU) als monstro simile (nach Pufendorfs Beschreibung des Heiligen Römischen Reichs) aus juristischer Sicht in Zukunft eher noch verstärkt zutreffen dürfte - bedingt durch die Kombination aus der expliziten Nicht-Staatlichkeit Europas, vor allem im Nachgang des gescheiterten Verfassungsvertrags, und den zunehmend komplexer werdenden intergouvernementalen Regelungsmechanismen. Umso mehr erweise sich die Nahtstelle zwischen europäischer Entwicklung und binnenstaatlichem Verfassungsrecht als Orientierungspunkt der deutschen staatsrechtlichen Diskussion um die Zukunft der EU. Im Kern stünde dabei die höchstrichterliche Maßgabe, dass die staatliche Souveränität der Bundesrepublik selbst ein Schutzgut des Grundgesetzes darstelle, wie bereits durch das Maastricht-Urteil skizziert und in der Lissabon-Entscheidung konkretisiert. Die daraus unter Verweis auf Art. 146 GG gezogene Konsequenz, nach der eine deutlich über das derzeitige Maß hinausgehende europäische Integration neues Verfassungsrecht voraussetze, führe in Teilen zu merkwürdigen Stilblüten (etwa dem Vorschlag einer Wahl des kommenden Bundestages als verfassunggebende Versammlung) und ideologischen Grabenkämpfen, obwohl eine pragmatischere und weniger zugespitzte Diskussion dem Gegenstandsbereich wesentlich zuträglicher wäre. Zentral sei hier die Bewältigung versteckter Dissense, die zwischen den Mitgliedstaaten etwa beim Vertrag von Maastricht im Bereich der Währungsunion - bestünden und erkennbar fortbestehen; diese seien kaum durch institutionelle Planspiele zu bewältigen. Hinzu trete ein zurzeit deutlich werdendes Unverständnis der ,Völker Europas“ untereinander, das vermeidbare Animositäten verursache und dem hinter Vorhaben wie der gemeinsamen Währung stehenden Ziel der Annäherung diametral widerspreche. Schließlich gelte, dass nicht nur die europäischen Einrichtungen, sondern auch die nationalstaatlichen Organe kaum zufriedenstellend funktionierten - insbesondere mit Blick auf die parlamentarische Kontrolle europäischer Politik. Die daraus zu ziehende Konsequenz bestehe mithin in einer differenzierteren und sensibleren Form der Debatte, die ergebnisoffen und zugleich zielorientiert zu führen sei - mit Blick auf die aktuellen Fragen der europäischen Politik ebenso wie hinsichtlich der Zukunft der EU und den daraus für den Nationalstaat erwachsenden Folgen.

Auf diese programmatische Einführung folgte ein Beitrag von Horst Dreier (Würzburg), der sich unter dem Titel „Auf dem Weg zum europäischen Bundesstaat?" aus rechtstheoretischer Sicht den skizzierten Problemen zu nähern suchte und dabei vor allem das Thema der Staatswerdung Europas und deren verfas- 
sungsrechtliche Voraussetzungen in den Mittelpunkt stellte. Die Ausführungen gründeten in der am amerikanischen und deutschen Beispiel diskutierten Frage, welche historischen Bedingungen Bundesstaaten hervorbringen. Im Hintergrund stünden stets einschneidende Veränderungen, die den qualitativen Sprung von einem Staatenbund zur Föderation ermöglichen und befördern. Die EU, bislang eher das Resultat eines kontinuierlich voranschreitenden Prozesses, sei zwar Krisen, aber keinem derartigen Schnitt ausgesetzt gewesen. Da sich dieser Prozess mit einem grundsätzlich vom Nationalstaat auf die europäische Ebene gerichteten Souveränitätstransfer verbinde, sei es allerdings durchaus vorstellbar, dass ein Bundesstaat auch inkrementell verwirklicht werden könne. Daraus entwickelte Dreier anhand des auf Zenon zurückgehenden Haufen-Paradoxons die Frage, ob die Schwelle der (Bundes-)Staatswerdung in einem solchen Prozess identifizierbar sei und ob das Grundgesetz diesbezüglich überhaupt eine klare Grenze definieren könne. Teile der Staatsrechtslehre verneinten dies, während andere, darunter Peter M. Huber und Udo Di Fabio (wie Dreier anhand entsprechender Vorträge aus dem Jahr 1993 zum Maastrichter Vertrag darlegte) zu dem Ergebnis kämen, dass Art. 79 Abs. 3 GG die souveräne Staatlichkeit schütze und darin eine unüberwindbare Hürde für einen europäischen Bundesstaat zu sehen sei. Einig wäre man sich nur darin, dass Art. 23 GG und der Vertrag von Maastricht eine solche qualitative Veränderung nicht dargestellt haben. Daran wiederum schließe die in der Einleitung des Panels bereits angesprochene Diskussion über die Rolle und Funktion von Art. 146 GG an, dessen vielfältige Interpretationen nach der Wiedervereinigung von der Obsoleszenz über den Begriff „,verfassungswidriges Verfassungsrecht" bis hin zum europapolitischen Allheilmittel reiche. Der Vortrag schloss mit dem Hinweis, dass diese Diskussion durch die Lissabon-Entscheidung des Bundesverfassungsgerichts insofern beendet wurde, als eine (der entsprechenden Passage aus dem im Jahr 2000 veröffentlichten Grundgesetz-Kommentar des Vortragenden nahezu wortgleiche) Formulierung Eingang in die Begründung fand, derzufolge die gliedstaatliche Einbindung der Bundesrepublik in eine europäische Föderation einer Verfassungsneuschöpfung gemäß Art. 146 GG bedürfe.

Da der zweite Vortrag in diesem Panel, den Udo Di Fabio unter den Titel „Die Unvollständigkeit Europas“ stellte, krankheitsbedingt vertagt werden musste, summierte Joachim Wieland (Speyer) als nächster Redner und discussant die Debatte anhand einer Reihe von Thesen. Danach sei zunächst an die europäische Mittellage Deutschlands zu erinnern, die in Verbindung mit seiner demographischen und wirtschaftlichen Bedeutung sowie den einschlägigen historischen 
Erfahrungen zu einer diskursiven Besonderheit führe, die die europapolitische Debatte in vielfacher Hinsicht präge und gleichzeitig erschwere. Dennoch könne man der Frage nach dem ,wohin“ kaum entkommen; Europa habe nur dann eine Verwirklichungschance, wenn eine (politische) Erzählung den Sinn der Integration darlege und dies mit klaren Zielvorstellungen verbinde. Diese müssten sowohl auf „mehr Europa“ als auch auf ein „besseres Europa“ (Hesse) drängen, allerdings kaum über eine maßstabsvergrößerte Anwendung der deutschen Vorstellung von Bundesstaatlichkeit. Einheitlich erkennbar sei in bestehenden Föderationen hingegen eine Entwicklung in Richtung wachsender Zentralisierung, die mithin auch die EU prägen dürfte. Die Währungsunion etwa dränge auf eine politische Union, der man sich nicht verwehren dürfe, da die notwendige politische Handlungsfähigkeit für die europäische Ebene sicherzustellen sei. Dies zu verwirklichen sei in Deutschland zwar durch Art. 146 GG möglich, allerdings (im Gegensatz zu der von Peter M. Huber geäußerten Einschätzung) nicht durch eine Teilrevision des Grundgesetzes, sondern ausschließlich im Zuge einer vollständigen Verfassungsneuschöpfung. Dabei sei mit Blick auf das Bundesverfassungsgericht und seine Entscheidungen stets zu beachten, dass auch institutionelle Interessen insofern eine Rolle spielen könnten, als das Gericht bei fortschreitender Integration durchaus Kompetenzverluste zu befürchten habe. Nicht zuletzt deshalb sei die Diskussion in Deutschland stark staatsrechtlich geprägt, obwohl die EU eigentlich und maßgeblich als politisches Projekt zu kommunizieren und vermitteln wäre. Daraus wiederum ergebe sich schließlich ein Bedarf an (europa-)politischer Führung, die über das gegenwärtig erkennbare Maß weit hinausgehen müsse.

Die nachfolgende Diskussion griff eine Reihe der angesprochenen Fragen auf, darunter die nach der europäischen Finalität, der Notwendigkeit eines dahingehend offeneren politischen Diskurses und dem sich damit wiederum verbindenden Bedarf an politischer Führung. Dies wurde ergänzt um eine Erörterung des benannten Desiderats einer ,europäischen Erzählung“ und deren konkreter Ausprägung, u.a. mit Blick auf die sich daraus ergebenden Anforderungen an die nationalstaatlichen wie die europäischen Organe.

\section{Wirtschaftswissenschaftliches Panel}

Das nachfolgende ökonomische Panel wurde im Wesentlichen von László Csaba (Budapest) und Bruno S. Frey (Zürich/Warwick) bestritten, da Sir Tony Atkinson (Oxford) und Werner Abelshauser (Bielefeld) gesundheitsbedingt nur durch knappe Aufrisse vertreten waren, in deren Rahmen sie einerseits eine dem enge- 
ren wirtschaftswissenschaftlichen Verständnis europäischer Politik zur Seite gestellte sozialpolitische Argumentationslinie skizzierten und andererseits unter dem Thema „Deutschland, Europa und die Weltwirtschaft: Inspektionseffekte der Finanzmarktkrise“ auf die historische Entwicklung, die Bedeutung und die Prägung von bzw. durch Wirtschaftskulturen abstellten.

In seinem einführenden Beitrag verwies László Csaba auf die Zugänge der Wirtschaftswissenschaften zur europapolitischen Diskussion und unternahm zunächst eine grundsätzliche tour d'horizon zur Rolle der (politischen) Ökonomie der EU in der gegenwärtigen disziplinären Auseinandersetzung. Das Thema sei in den universitären Curricula und auch in den Programmen der einschlägigen Verlagshäuser kaum vertreten; auch sei unter den Veröffentlichungen in bedeutenden (meist amerikanischen) journals der Wirtschaftswissenschaften, also den zentralen Organen der disziplinären Debatte, der Komplex Europa/EU im Allgemeinen und das europäische Verhältnis zum Nationalstaat im Besonderen in nur sehr wenigen Fällen präsent. So beschäftige sich etwa das renommierte Quarterly Journal of Economics auf interessante Weise mit Adipositasfragen, Scheidungsgründen, ethnischer Diskriminierung oder der Rolle von körperlicher Schönheit auf dem Arbeitsmarkt, in den vergangenen Jahren aber nicht ein einziges Mal mit den sich aus der europäischen Krise ergebenden Fragen. Gleichzeitig erwiesen sich diese in der Realität aber als eminent bedeutsam, stünden sie doch im Mittelpunkt der öffentlichen und veröffentlichten Diskussion, nicht nur in Europa selbst. Csaba verwies auf die paradoxe Situation, die sich damit verbinde, einer Disziplin zuzugehören, die zu einer zentralen (und für das Fach äußerst relevanten) Frage der aktuellen Politik sehr wenig zu sagen habe.

Im Anschluss entwickelte er fünf programmatische Thesen, die eine den Namen verdienende ökonomische Diskussion des laufenden Europäisierungsprozesses und seiner Formen, Funktionen und Folgen anleiten könnte. Zunächst sollte sich die Debatte auf die Rolle von Fiskal- und Geldpolitiken im europäischen Gefüge richten, da einerseits die Europäische Zentralbank (EZB) ihr Aufgabenspektrum in den vergangenen Jahren deutlich über das eigentliche Ziel der Gewährleistung von Preisstabilität hinaus erweitert habe und andererseits die fiskalpolitischen Verteilungsentscheidungen sich kaum für technokratische Steuerungsprozesse eigneten, sondern im Kern Gegenstand (demokratischer) Diskussion sein müssten. Insofern sei kritisch zu diskutieren, ob die derzeit entwickelten fiskalischen Kontrollmechanismen diese fundamentale Unterscheidung nicht gerade vernachlässigten und welche Konsequenzen sich daraus ergeben könnten. Zum zweiten sei die vorgesehene Überwachung von etwa 6.000 Finanzinstituten und den be- 
stehenden nationalstaatlichen Einrichtungen durch die EZB im Rahmen einer europäischen Bankenunion zu diskutieren, da hier einerseits tiefe Eingriffe in die Wirtschaftssysteme der Mitgliedstaaten wahrscheinlich werden und andererseits die Sinnhaftigkeit der Delegation einer solch immensen Aufgabe an eine einzige Einrichtung schon kapazitäts- und kompetenzbezogen in Zweifel stünde. Drittens sollte die Frage der wirtschaftspolitischen Koordination hinsichtlich der damit verfolgten Zielsetzungen und der dazu eingesetzten Mittel wesentlich ausdifferenzierter diskutiert werden, unter anderem mit Rücksicht auf das Verhältnis von technokratischem Krisenmanagement und demokratischer balance of power. Viertens wären die Wirtschaftswissenschaften gut beraten, sich in die Diskussion um die Finalität Europas einzuschalten und aufzuzeigen, welche Form der Krisenbewältigung zu den erwünschten outcomes beitragen könnte, etwa mit Blick auf den erkennbaren wirtschafts- und ordnungspolitischen Reformbedarf. Schließlich müsste, gerade unter den benannten disziplinären Voraussetzungen, der erkennbare drift zwischen öffentlicher Meinung und Elitenverhalten im Rahmen der europäischen Krise erörtert werden, um zu klären, ob und inwiefern er die europäische Entwicklung gefährden könnte und wie dem begegnet werden sollte. Von einer Vorstellung eigener Beiträge (vgl. hierzu die jüngeren Hefte der ZSE) sah Csaba ab, verwies aber auf die darin kenntlich gemachten Differenzen zwischen den westeuropäischen Mitgliedstaaten und den Neumitgliedern aus Mittel- und Osteuropa. Demzufolge verdecke die häufig in den Vordergrund gerückte Nord-Süd-Dichotomie weitere Unterschiede und Disparitäten, die eine kompetente wirtschafts- und finanzpolitische Diskussion nicht ausblenden sollte.

Diese Programmatik griff Bruno S. Frey in seinem Vortrag zum Thema „Die Turbulenzen in der Eurozone und die Zukunft der WWU - ein Blick von außen“ eher implizit auf, indem er einen gleichsam idealtypischen Entwurf nicht nur wirtschaftspolitischer, sondern auch allgemein anwendbarer Steuerungsmöglichkeiten in den Raum stellte. Zunächst sei in der Diskussion zwischen europäischen Problemen und Problemen der EU deutlicher zu unterscheiden, da sich diese zwar gegenseitig bedingten und beeinflussten, aber gewiss nicht deckungsgleich seien. Letztere stellten insbesondere das Ergebnis eines ab ovo falsch angelegten Integrationsprozesses dar, der die Herausforderungen, denen er sich heute stellen muss, selbst geschaffen habe. Die Verbesserungsfähigkeit des Integrationsprozesses suchte Frey anhand von drei Thesen aufzuzeigen. Zum einen sei die EU auf der Grundlage von Nationalstaaten gedacht und vollzogen, was zwar der territorial-gebietskörperschaftlichen Realität entspräche, aber gerade deshalb keinen grundlegend neuen Ansatz darstelle und zahlreiche diskussions- 
würdige Auswirkungen zeitige. So sei zwar das europäische Friedensprojekt anzuerkennen, doch sollte man in der EU nicht die Ursache, sondern das Ergebnis der friedlichen Orientierung vor allem Deutschlands und Frankreichs nach dem Zweiten Weltkrieg sehen. Die EU selbst habe kaum zum Frieden beigetragen, was sich am deutlichsten bei einem Blick auf Nordirland, das Baskenland und den Balkan, aber eben auch bei der Bewältigung der Euro-Krise zeige. Die wirtschaftliche Integration hingegen sei durch Bürokratisierung, Harmonisierung, Uniformierung und das Erfordernis einer einheitlichen Übernahme des acquis communautaire geprägt, die ein „weiter so“ wenig zielführend erscheinen ließen. Allerdings gebe es, so die zweite These, eine Alternative insofern, als man sich in Zukunft vor allem an den erkennbaren Problemstellungen ausrichten sollte, wobei die territoriale Ausrichtung der verfolgten Lösungsansätze der Reichweite der Problemstellungen folgen müsse. Es gehe um die Schaffung flexibler, weniger an bestehenden Grenzen ausgerichteter und vielmehr ,problem-endogener" politischer Einheiten, die sich konkreten Lösungsansätzen widmen würden. Mitglieder solcher Einrichtungen wären Regionen, Gemeinden, Gemeindeteile oder auch Individuen. Sie sollten über fiskalische Kompetenzen verfügen, demokratisch verfasst sein, klare Ein- und Austrittsregeln formulieren und nicht oktroyiert werden, sondern in bottom-up-Prozessen entstehen. Ein Beispiel sei die Regulierung des Bodenseeraums, die auf gebietskörperschaftsübergreifende, einheitliche Problemstellungen zu reagieren suche. Schließlich sei es keinesfalls zu spät, solche Steuerungsformen schrittweise einzuführen, da eine gewisse endogene Problemorientierung auch am Anfang des europäischen Integrationsprozesses gestanden habe und eine Schaffung derartiger politischer Einheiten über ein (insofern erstrebenswertes) Europa der unterschiedlichen Geschwindigkeiten auch im Rahmen der bestehenden EU denkbar wäre. Voraussetzung sei ein gewisses Maß an Flexibilisierung, Dynamisierung und Entbürokratisierung des Integrationsprozesses, um in dessen weiterem Verlauf eine produktive Vielfalt von problemadäquaten Ansätzen zu ermöglichen.

Der damit skizzierte Entwurf eines wesentlich ausdifferenzierteren Europäisierungsprozesses wurde in der nachfolgenden Diskussion zwar umfassend gewürdigt, unterlag aber einer Reihe von Kritiken. So verwiesen Vertreter unterschiedlicher gebietskörperschaftlicher Ebenen darauf, dass entsprechende funktionale Einrichtungen doch sowohl im Rahmen der Nationalstaaten als auch grenzüberschreitend bereits existierten, während andere Diskutanten für die europäische Ebene auf unterschiedliche Teilprojekte (Schengen, Eurozone, verstärkte $\mathrm{Zu}-$ sammenarbeit) und externe Kooperationsformen verwiesen, um die Validität der 
angegriffenen Prämisse einer ausschließlich nationalstaatlichen Orientierung in Zweifel zu ziehen. Schließlich wurde auch die Frage aufgeworfen, welcher Instanz im Rahmen eines solch ausdifferenzierten Steuerungsmodells die (verbindliche) Definition der zu lösenden Probleme zuzuordnen sei.

\section{Sozialwissenschaftliches Panel}

Das sich am frühen Nachmittag anschließende sozial- und hier vor allem politikwissenschaftliche Panel wurde von Joachim Jens Hesse (Berlin) eingeleitet, der zunächst auf die im Rahmen staats- und europawissenschaftlicher Fragestellungen gewachsene Bedeutung der Sozialwissenschaften verwies. Sie hätten vor allem empirisch-analytisch und methodisch den Nachbardisziplinen gegenüber beträchtlich aufgeholt und ebneten den Weg hin zu einem produktiven interdisziplinären Ansatz. Diese Kompetenz - das Ergebnis endogener Entwicklungen und exogenen Handlungsdrucks - habe über eine konstruktive Verbindung von grundlagen- und anwendungsorientierter Forschung inzwischen zahlreiche empirisch gesättigte und methodisch anspruchsvolle Untersuchungen zum Verhältnis von Nationalstaat und EU hervorgebracht. Es seien diesbezüglich vor allem drei Arbeitsfelder mit beträchtlichem Ertrag erkennbar: So wurde die Erörterung von nationalstaatlichen wie europäischen Willensbildungs- und Entscheidungsprozessen zunehmend ausdifferenziert, die Gestaltung eines für den Integrationsprozess funktionalen, institutionell-organisatorischen Rahmens (in unitarischen wie föderalstaatlichen Systemen) konzipiert und auf komplementäre Veränderungen von Verfahrens- und Verhaltensweisen der Akteure verwiesen. Dabei gelte die Aufmerksamkeit im ersten Arbeitsfeld neuen oder veränderten Formen von Partizipation, Legitimation und Identitätsbildung, die den Bedürfnissen nach Vergewisserung und Rückversicherung zu entsprechen suchen und häufig kulturell disparaten Kontexten entspringen, während institutionell-organisatorische catchall-Kategorien und übermäßig aggregierte Analyseeinheiten (wie die des „Mehrebensystems") heruntergebrochen wurden, wobei der Zuwachs an konkreten handlungsorientierten Untersuchungen besonders erfreulich sei (unter Einschluss einer gewissen analytischen „Wiedergeburt“ des Vergleichs von Regierungs- und Verwaltungssystemen - binnenstaatlich, mit Blick auf die europäische Entwicklung und im Abgleich von und mit anderen Formen der Regionalorganisation, wie der ASEAN oder dem MERCOSUR). Schließlich werde mit Blick auf die eingesetzten Verfahren deutlich, dass der erkennbare Handlungsbedarf und die vorgehaltenen Handlungskapazitäten zunehmend auseinanderzufallen drohen, mit dem Ergebnis häufig suboptimaler Lösungen. Hinsichtlich der Möglichkeit 
eines britischen EU-Austritts böte sich die Chance, die Vorschläge der Londoner Regierung zu einer Reform der Union eher aufzugreifen denn abzuweisen, zumindest dann, wenn sich damit eine konsequente Überprüfung der europäisch wie nationalstaatlich wahrgenommenen Aufgabenbestände verbinde. Diesem ersten Schritt einer den Namen verdienenden Aufgabenkritik könnten sich dann funktionalreformerische Bemühungen anschließen, bevor auf dieser Basis ggf. organisationsstrukturelle Konsequenzen gezogen würden. Für entsprechende Überprüfungen des europäischen und nationalstaatlichen Aufgabenbestandes seien die anwendungsorientierten Sozialwissenschaften prädestiniert.

Der folgende Vortrag von Uwe Wagschal (Freiburg) zum Thema „Staatstätigkeit und europäische Identität" nahm einige dieser Zugänge auf und suchte vor allem die output-Legitimation der EU zu quantifizieren. Auf der Basis einer grundsätzlichen Bestandsaufnahme der Zielsetzungen europäischer Politik unter Rückgriff auf „Staatstätigkeitsfelder“ suchte er die Performanz der EU anhand einer Reihe von Indikatoren zu bewerten. Zunächst wandte sich auch Wagschal der EU als Friedensprojekt zu und konstatierte seit Gründung wenigstens 14 gewalttätige Konflikte (darunter sieben Kriege), die von Mitgliedstaaten geführt wurden, während sich die innereuropäische Pazifierung auch durch die Theorie des demokratischen Friedens gut erklären lasse. Zudem stehe die von der Friedens- und Konfliktforschung als maßgeblich stabilisierender Faktor identifizierte Abwesenheit eines Überhangs junger Männer in der demographischen Struktur der Mitgliedstaaten höchstens indirekt mit der EU in Verbindung. Andererseits sei der stark ausgeprägte zwischenstaatliche Handel, der anerkannt friedensstiftende Wirkungen entfalte, überwiegend der europäischen Integration zuzuschreiben, sodass der Befund des „friedenssichernden Europas“ in der Summe nicht gänzlich von der Hand zu weisen sei. Als zweites Handlungsfeld wurde die wirtschaftliche Entwicklung herangezogen und über einen Vergleich der EU-15 mit anderen OECD-Staaten für die Jahre 1995 bis 2011 operationalisiert. Danach wiesen die EU und ihre Mitgliedstaaten, gemessen an Indikatoren wie Staatsausgaben, Wirtschaftswachstum oder Inflation, in Teilen durchaus unterdurchschnittliche Werte auf. Dieser Befund bleibe bestehen, wenn sich die Analyse auf die Zeit zwischen 2004 und 2011 beschränke und die EU-27 umfasse, mithin den catch-up-Effekt der neuen Mitgliedstaaten besonders betone. Zum dritten richtete sich die Untersuchung auf den Bereich der Staatsverschuldung, den man bereits durch den Stabilitäts- und Wachstumspakt europäisch zu regeln versuchte. Hier stellte Wagschal eine hohe Quote an Regelverstößen fest, was auf eine fundamentale Nichtakzeptanz der Regeln und einen strategischen Verschul- 
dungsanreiz im Rahmen der EU verweise. Darüber hinaus gewöhne man sich im Rahmen des sog. flypaper-Effekts an einmal gewährte Subventionszahlungen, sodass strukturelle Regelungsdefizite zu dauerhaften Kosten führten. Andererseits zeitige die gegenwärtige Krise etwa in Deutschland deutlich positive Effekte, unter anderem durch Zinsgewinne für die öffentliche Hand und für private Kreditnehmer, sodass man argumentieren könne, die Hilfen für die überschuldeten Staaten seien lediglich eine „kleine Gebühr“, die den benannten Vorteilen zuzuordnen wäre. Verbunden mit einer mangelnden input-Legitimation und der durch dauerhafte Regelverletzung untergrabenen Prozess-Legitimation ergebe sich insofern ein grundlegendes Problem, als der Staat bzw. die Union ihr Handeln zunehmend nicht mehr durch den erreichten output zu rechtfertigen vermögen. Dies spiegele sich in den abnehmenden Quoten affektiver wie utilitaristischer Zustimmung für das europäische Projekt im Rahmen von EurobarometerUmfragen und Volksabstimmungen mit europapolitischem Inhalt.

Den zweiten Vortrag im Rahmen dieses Panels hielt Fritz W. Scharpf (Köln) unter dem Titel „Was soll und kann die Europäische Union?“; er ist im vorliegenden Heft dieser Zeitschrift dokumentiert und soll daher nur kurz skizziert werden. Scharpf ging von einem grundlegenden Spannungsverhältnis zwischen zwei europapolitischen Maximen aus, die sich einerseits auf den Wert der europäischen Integration an sich, andererseits auf eine zweckrationale Vorstellung von Europa beriefen. Dies führe dazu, dass man durch verstärkte Integration der verminderten Effektivität und Legitimität nationalstaatlicher Politik zu begegnen suche, dabei aber Probleme schaffe, die die EU nicht unbedingt selbst zu lösen vermag. Grund dafür sei der hohe Konsensbedarf bei ausgeprägter Heterogenität; Konsens werde häufig durch Entpolitisierung, Richterrecht oder Formelkompromisse erzeugt. Dadurch werde die Lernfähigkeit der EU eingeschränkt, einmal getroffene Entscheidungen könnten kaum revidiert werden. Die Währungsunion stelle dabei einen Extremfall dar, weil die monetäre Überintegration nicht mit entsprechenden Instrumenten in anderen Bereichen flankiert wurde. Die Angleichung der Nominalzinsen führte zunächst zu unterschiedlichen Wachstumsraten und in der Folge von Reformpolitiken zu auseinanderdriftenden Lohnstückkosten, womit sich mittelfristig divergierende reale Wechselkurse (und unterschiedliche Wettbewerbsfähigkeit) verbanden. Dies habe zu starken Leistungsbilanzdefiziten geführt, die sich in der Währungsunion bis zur LehmanFinanzkrise 2008 ungehindert entwickeln konnten. Da die Defizitländer von Krediten abhängig waren, traf sie der Schock der Kreditverknappung am härtesten und führte in eine tiefe Staatsschulden-, Wirtschafts- und Sozialkrise. Hohe 
Staatsverschuldung war hingegen, und entgegen der verbreiteten Meinung, nicht Ursache der Entwicklung, doch bilde dieser Trugschluss die Grundlage der ,Rettungspolitik“, die als Insolvenzregime nicht demokratisch legitimiert sei. Die Krisenbewältigung treibe die erkennbare Zentralisierung weiter voran und sehe eine Kontrolle der nationalen Mikropolitiken vor, statt über eine gegebenenfalls notwendige Dezentralisierung zu diskutieren. Dafür werden Verluste demokratischer Legitimität in Kauf genommen, was zu eskalierenden Konflikten zwischen den Mitgliedstaaten führen könne. Scharpf kam zu einem ernüchternden Ergebnis: „Die EU tut, was sie weder soll noch kann.“

In seinem zusammenfassenden Kommentar ging Manfred G. Schmidt (Heidelberg) kurz auf konjunkturelle und strukturelle Demokratiedefizite der EU ein, die trotz aller nationalstaatlicher Legitimation Platz griffen. Jenseits der zurzeit vorherrschenden (aber reformierbaren) Art, europäische Politik zu betreiben, liege in den benannten output-Defiziten, der fehlenden „Erfahrungsgemeinschaft“ sowie den heterogenen Sprachen und Identitäten Europas ein strukturelles legitimatorisches Problem, das nicht einfach zu überwinden sei, aufgrund der angesprochenen Verflechtungen und Herausforderungen allerdings dringend überwunden werden müsse. Gleichzeitig sei von allzu pessimistischen Einschätzungen abzuraten, da die erkennbaren Probleme durchaus mit einer grundsätzlichen Zustimmung gepaart seien, der Souverän sich also zwar geneigt zeige, mit der konkreten Funktionsweise der EU aber unzufrieden sei - wie auch im Fall der demokratischen Systeme der Nationalstaaten. Zudem gäbe es in einigen Politikfeldern, etwa im Bereich der Lohn- und Sozialpolitik, aber in gewissem Umfang auch bei der Fiskalpolitik, durchaus noch beträchtlichen Handlungsspielraum auf nationalstaatlicher Ebene. Zugleich sei bei den Eliten eine ideologische (und wirtschaftsfremde) Verständigung auf das europäische Projekt zu beobachten, während der demos sich in vielerlei Hinsicht interessenfern verhält - in den bislang profitierenden Krisenstaaten deutlich europafeindlich ausgeprägt, in den Geberstaaten weitgehend apathisch.

Im Rahmen der Aussprache wurde deutlich, dass die These von einer Bedeutungssteigerung sozialwissenschaftlicher Zugänge zu den im Rahmen des Kolloquiums verhandelten Themen bestätigt werden konnte. Vor allem die Ergänzung der normativ geprägten Zugänge der Staatsrechtslehrer durch funktional ausgerichtete sozialwissenschaftliche Analysen erwies sich für die Erklärung einiger der gegenwärtig zu verzeichnenden europäischen Turbulenzen als produktiv. Hinzu trat eine Diskussion über die Wahrnehmung der europäischen Krise durch den Souverän und die sich damit verbindenden Herausforderungen. 


\section{Geschichts- und kulturwissenschaftliches Panel}

Die Einführung in das abschließende Panel übernahm Friedrich Wilhelm Graf (München), der den kulturwissenschaftlichen Forschungsstand zur Europadiskussion als weder besonders ausgeprägt noch besonders augenfällig kennzeichnete. Zwar seien bereits in den 1920er Jahren im Nachgang des Ersten Weltkriegs einige wenige europabezogene Diskussionen erkennbar gewesen, etwa durch Ernst Troeltschs Diktum von der „europäischen Kultursynthese“ aus calvinistischen und katholischen Traditionen, aber erst seit den 1960er Jahren könne man deutlich unterscheidbare Europakonzepte nachweisen. Mit Blick auf die gegenwärtige Diskussion verwies Graf auf das die europäische Entwicklung dominierende Phänomen einer zunehmenden Zentralisierung und Integration bei gleichzeitig voranschreitender lebensweltlicher Diversifizierung und weltanschaulicher Pluralisierung. Die dabei zu beobachtende Multiplizierung von Sinnund Orientierungsanbietern sei keineswegs nur ein Ergebnis von Migration und kultureller Öffnung, sondern auch ein Resultat innereuropäischer Entwicklungen; so verzeichne man etwa einen starken Anstieg bei der Zahl der organisierten christlichen Gemeinschaften. Bei der Suche nach den mehrfach angesprochenen „europäischen Narrativen“ oder „,sinnstiftenden Erzählungen“ sollte man diese vorhandene Vielfalt nicht außer Acht lassen.

Im ersten der beiden materiellen Vorträge dieses Panels suchte Dieter Langewiesche (Tübingen) unter dem Titel „Föderalstaatliche Traditionen und europäischer Handlungsbedarf" aus historischer Perspektive zwei Fragen zu beantworten, die für die gegenwärtige europäische Entwicklung von Gewicht sein dürften: Was bedeutete Föderalismus in den europäischen und amerikanischen Staatsbildungsprozessen? Und: Wie verlief die Identitätsbildung in Föderalstaaten? Zum erstbenannten Komplex wurde zunächst (und in Ergänzung der Ausführungen von Horst Dreier) konstatiert, dass der Übergang vom Staatenbund zum Bundesstaat stets mit Gewalt - Revolution oder Krieg - verbunden war. Dies werde durch die Rede von der „Pfadabhängigkeit“ häufig in den Hintergrund gestellt, doch hätten etwa die bedeutenden Weichenstellungen der deutschen Föderalstaatlichkeit 1806, 1848-49, 1867-71, 1919 und 1945-49 - ausnahmslos im Kontext von Gewalterfahrungen gestanden. Auch hätten die Schweiz und die USA ähnliche Erfahrungen gemacht; die Pfadabhängigkeit bestehe mithin vor allem darin, dass im Zuge von Krieg und Revolution die historischen Pfade durchbrochen werden. Dies gelte auch für die Gründung der EU, nicht aber für ihre weitere Entwicklung - vor allem im Rahmen der Integration der mittel- und osteuropäischen Staaten. Hier sei eine harte historische Regel widerlegt worden, sodass es kein 
Vorbild geben könne. Die Pfadbildung in Europa scheine heutzutage eher durch andersartige krisenhafte Zuspitzungen zu geschehen. Zugleich sei deutlich zwischen Föderalismus und Supranationalität zu unterscheiden, da ersterer ein historisches Resultat einer langen Entwicklung „zusammengesetzter Staatlichkeit“ (res publica mixta) darstelle, die im 19. Jahrhundert zunehmend unter Reformdruck geriet. Die Überwindung oder Transformation des zusammengesetzten Staates habe zu unterschiedlichen Denk- und Handlungstraditionen in den EUMitgliedstaaten geführt, sodass von einer Anwendung des Föderalismus-Begriffs im Zusammenhang mit der europäischen Entwicklung eher abzuraten sei - insbesondere aufgrund der starken Prägung der Staatswerdung im 19. Jahrhundert durch das wirkmächtige Konzept der Nation. Die europäische Supranationalität hingegen suche genau dieses Konzept zu vermeiden. Insofern könnte sich der eigentlich überwundene Begriff der zusammengesetzten Staatlichkeit als produktiver erweisen.

Mit Blick die identitätsbildende Wirkung von Föderalstaaten verwies Langewiesche zunächst auf die herrschende Meinung, derzufolge die Staatswerdung Europas (samt eines vollständig legislativ und fiskalisch befugten Europäischen Parlaments) ein Staatsvolk voraussetze, das derzeit bekanntlich nicht gegeben sei. Bis dahin bliebe die einzige Legitimationsbasis die nationalstaatliche Ordnung. Aus historischer Sicht sei allerdings festzuhalten, dass die Ethnogenese der Staatsbildung in der Regel nachfolgt - nicht das Volk schuf den Staat, sondern die europäischen Staaten definierten die „Völker Europas“. Gleichwohl beruhten die nationalstaatlichen Mythen Europas nach wie vor auf der umgekehrten Sichtweise, was sich als schwere Hypothek für die EU erweise. Dabei gingen gerade in Deutschland, Österreich und der Schweiz Nation und Staat historisch überwiegend getrennte Wege; erst kriegerische Auseinandersetzungen konnten dem entgegenwirken. Die breite gesellschaftliche Legitimation ging der Staatsbildung nicht voraus, sie erwuchs ex post aus ihr; es handelte sich stets um Elitenprojekte. Insofern stelle dies mit Blick auf die EU keinen plausiblen Hinderungsgrund oder gar die Ursache für ein dauerhaftes Demokratiedefizit dar. Allerdings müsse die Staatsbildung zu einer verbesserten Lebenswirklichkeit führen und Partizipationsmöglichkeiten eröffnen, um eine breite gesellschaftliche Anerkennung zu erzeugen. Ob die Katalysatorfunktion der Gewalterfahrung im Fall Europas dauerhaft durch die krisenhafte Zuspitzung politischer und wirtschaftlicher Fragen ersetzt werden kann und deshalb die Staatswerdung ebenso befördern könne, bleibe abzuwarten. 
Der zweite historisch ausgerichtete Vortrag, gehalten von Harold James (Princeton), befasste sich mit der Frage nach der „Europäische[n] Union als Vorbild?““, was gleich zu Beginn der Ausführungen als fast unlösbares Problem charakterisiert und in die Frage nach historischen Vorbildern für die EU abgewandelt wurde. In diesem Zusammenhang werde, vor allem mit Blick auf die Frage der fiskalpolitischen Integration, häufig auf die Staatswerdung der USA und die dabei erfolgte Übernahme der Kriegsschulden der Einzelstaaten durch den Bund im Jahr 1790 verwiesen, die hier erst nach der politischen Vereinigung im Jahr 1788 vollzogen wurde. In den USA waren insbesondere die Staaten Neuenglands stark verschuldet, während in Virginia, dem Heimatstaat vieler hochrangiger Bundespolitiker, kaum Verbindlichkeiten anhängig waren. Insofern mussten bestimmte Garantien vereinbart werden: Zum einen wurden Schuldenübernahmen im Gesamtvolumen begrenzt und zum anderen konnte Virginia den Hauptstadtdiskurs gegen Philadelphia bzw. New York zu seinen Gunsten prägen. Die nun gemeinsamen Schulden wurden wiederum durch gemeinsame Einkünfte gedeckt, etwa über Zolleinnahmen des Bundes. Die Zölle bevorteilten allerdings die Nordstaaten und benachteiligten die Südstaaten, führten mithin zu Verteilungskämpfen und trugen in letzter Konsequenz auch zum Ausbruch des Amerikanischen Bürgerkriegs bei; die Eröffnung der für eine Fiskalunion notwendigen Einnahmequellen führten mithin zu neuen, schwerwiegende(re)n Konflikten. All dies und vor allem den darin dokumentierten Umgang mit Differentialen zwischen Geberund Nehmerstaaten sowie die daraus erwachsenden Differenzen sollte man bei der Frage der künftigen europäischen Entwicklung beachten, um nicht durch kurzsichtige Krisenreaktionen langfristig mehr Probleme zu schaffen als zu lösen. Schließlich sei auch die Schweiz als mögliches Vorbild für Europa zu nennen, sowohl mit Blick auf die demokratische Legitimation politischer Entscheidungen auf unterschiedlichen gebietskörperschaftlichen Ebenen als auch hinsichtlich des Umgangs mit Binnenkonkurrenz und Verteilungsprozessen.

In seiner zusammenfassenden Darstellung ließ Brendan Simms (Cambridge) die beiden Vorträge noch einmal Revue passieren und fügte eine Reihe von ergänzenden Beobachtungen hinzu. Einerseits seien weitere historische Vorbilder zu nennen, um die Diskussion über die Zukunft der EU zu befördern, darunter (als Negativbeispiel) das Heilige Römische Reich, das nicht an internen Konflikten, sondern externem Druck zugrunde ging, und das Vereinigte Königreich (als Positivbeispiel), das sowohl die Schuldenproblematik als auch sicherheitspolitische Anforderungen erfolgreich zu akkomodieren vermochte. Dies sei insofern relevant, als die EU heute eher dem Alten Reich als dem Vereinigten Königreich 
ähnele, wobei gerade (statt einem europäischerem Großbritannien) ein britischeres Europa hilfreich wäre. Andererseits sei die Vergemeinschaftung der Schulden in den Jahren 1788-1790 nur ein Aspekt der Staatswerdung der USA, der Aufbau von militärischen und außenpolitischen Kapazitäten und Kompetenzen auf Bundesebene traten als bedeutsame Entwicklungen hinzu. Nur so konnte das Übergewicht der Gliedstaaten umgekehrt werden, die wiederum aus Furcht vor Hegemonialansprüchen anderer states einer solchen Regelung zustimmten und darüber hinaus festlegten, dass neue Territorien nicht bestehenden Gliedstaaten zugeschlagen, sondern zu neuen Staaten des Bundes werden sollten. Schließlich sei daran zu erinnern, dass der europäische Einigungsprozess von Anfang an eine Antwort auf die deutsche Frage darstellte, sowohl in den 1950er Jahren als auch im Kontext der Wiedervereinigung. Da diese Frage nun abschließend beantwortet sei, bleibe abzusehen, ob das europäische Projekt heute auf vergleichbar treibende Kräfte zurückgreifen könne.

Die nachfolgende Diskussion richtete sich zunächst auf die Frage, ob eine etwaige deutsche Hegemonialstellung durch einen europäischen Bundesstaat befördert würde oder im Gegenteil der Verzicht auf weitere Integration zu einer dominanteren Stellung Deutschland im intergouvernementalen Europa führe. Zudem wurde angemerkt, dass bundesstaatliche Lösungen zwar im Regelfall mit Gewalterfahrungen einhergehen, ein europäischer Einigungskrieg historisch aber nicht erkennbar sei (mit Blick auf den Balkan etwa wurde auf die Notwendigkeit mehrfachen amerikanischen Einschreitens verwiesen). Damit in Verbindung stand schließlich die Diskussion darüber, ob solche Konflikte sich heute an anderer Stelle andeuteten und ob bzw. unter welchen Voraussetzungen krisenhafte Zuspitzungen wie die gegenwärtigen wirtschafts- und währungspolitischen Herausforderungen die benannte Regel von der Gewalt als „Motor der Staatswerdung" durchbrechen könnten.

\section{Zusammenfassung}

Die vielfältigen und punktuell durchaus kontrovers geführten Debatten zu den einzelnen Beiträgen der die Staats- und Europawissenschaften konstituierenden Disziplinen ließen erkennen, dass die gegenwärtige krisenhafte Entwicklung von fast allen Referenten und zahlreichen Diskutanten als eine Art von Wende- und Entscheidungspunkt wahrgenommen wurde, der die europäische Entwicklung prägen und wohl auch materiell beträchtlich verändern dürfte.

Während sich dies in der öffentlichrechtlichen Diskussion vor allem auf die Frage nach der Rolle der „Staatlichkeit“ des Grundgesetzes in der künftigen EU 
richtete, sahen die Wirtschaftswissenschaftler sich mit einer gewissen kritischen Zuspitzung insofern konfrontiert, als sie zwar auf eine längere Debatte über Vorund Nachteile einer Währungsunion verweisen konnten, für die weitere Entwicklung aber bislang nur wenige Handlungsalternativen aufzeigen konnten. Dem sucht man in Teilen, wie in der Diskussion dokumentiert, durch die Entwicklung stärker anwendungsorientierter, mithin empirisch basierter Untersuchungen zu begegnen. Die Sozialwissenschaften wiederum nehmen die Krise erkennbar zum Anlass, über Formen und Funktionen der EU wesentlich umfassender als bislang nachzudenken, zum einen aus der Perspektive der Leistungsfähigkeit und einer etwaigen Reformierbarkeit des europäischen politischen Systems, zum anderen mit Blick auf dessen demokratische Legitimation und Akzeptanz. Bei den sich daraus ergebenden Fragen nach einer möglichen Entwicklung hin zu einem europäischen Bundesstaat wiederum ließen die Historiker aufhorchen, die aus der Erfahrung mit Staatswerdungsprozessen zur Vorsicht mahnen und auf größere Problemstellungen verweisen, deren Berücksichtigung die tagesaktuellen Herausforderungen relativieren könnte.

Der mit dem Kolloquium ermöglichte interdisziplinäre Austausch erwies sich im Ergebnis nicht nur als höchst produktiv, er bot vielmehr auch einen willkommenen Anlass, die jeweiligen disziplinären Grundpositionen zu überprüfen und eine Reihe sonst häufig isoliert geführter Diskurse aufeinander zu beziehen. Im Juni 2013 wird das Gespräch in Berlin, dann unter Einbezug von Vertretern des politisch-administrativen Bereichs, fortgesetzt. Eine gesonderte Veröffentlichung der Erträge des Kolloquiums ist für Ende 2013 vorgesehen. 